\title{
Square-integrable representations of non-unimodular groups
}

\section{A.L. Carey}

In the last three years a number of people have investigated the orthogonality relations for square integrable representations of non-unimodular groups, extending the known results for the unimodular case. The results are stated in the language of left (or generalized) Hilbert algebras. This paper is devoted to proving the orthogonality relations without recourse to left Hilbert algebra techniques. Our main technical tool is to realise the square integrable representation in question in a reproducing kernel Hilbert space.

\section{Introduction}

If $G$ is a locally compact unimodular group and $L$ the left regular representation, then the square integrable representations of $G$ are the irreducible subrepresentations of $L$. It was shown by Godement $[5,6]$ and later more generally by Dixmier [2] that the Schur orthogonality relations hold for these representations. In the last few years non-unimodular groups have been investigated [3], [8], [11] and analogous results proved. The theorems are stated, as befits their generality, in the language of left (or generalized) Hilbert algebras. This tends to make the results rather inaccessible to the non-specialist.

The object of this paper is to derive the orthogonality relations for square integrable representations of non-unimodular groups by a method which avoids the use of left Hilbert algebras and which also says something different even for the unimodular case.

Received 11 March 1976. 
We pause for a definition.

DEFINITION 1.1. Let $X$ be a topological space and $H$ a Hilbert space whose elements are continuous functions from $X$ to $C$. We say $H$ is a reproducing kernel Hilbert space if there is a function $\phi: X \times X \rightarrow C$ such that

(i) the functions $\phi_{x}$ defined by

$$
\phi_{x}(y)=\phi(x, y)
$$

lie in $H$ for all $x$ in $X$,

(ii) for all $f$ in $H, f(x)=\left\langle\phi_{x}, f\right\rangle$.

The nomenclature derives from property (ii) which expresses the 'reproducing' property of the kernel $\phi$. Now let $G$ be a locally compact group with left Haar measure $d x$ and consider the left regular representation $L$ of $G$ in $L^{2}(G)$. Clearly, the objects of interest here are the minimal projections in the commuting algebra of $L$ (these are the minimal idempotents of the left Hilbert algebra). We show that every irreducible square integrable representation may be realised in a reproducing kernel Hilbert subspace of $L^{2}(G)$ and that the projection onto this subspace is given by a right convolution operator $R_{\phi}$, some $\phi \in L^{2}(G)$. The function $x, y \rightarrow \phi_{x}(y)=\phi\left(x^{-1} y\right)$ is found to be the reproducing kernel and this fact is used to derive the orthogonality relations.

Besides the notation introduced above, we will denote the modular function of $G$ by $\Delta$ and $L^{2}(G)$ will represent the Hilbert space defined by the functions on $G$ square integrable with respect to left Haar measure $d x$. The map $S$ defined by

$$
(S f)(x)=\overline{f\left(x^{-1}\right)} \Delta\left(x^{-1}\right)
$$

is easily shown to be a closed densely defined operator on $L^{2}(G)$ with adjoint

$$
\left(S^{*} f\right)(x)=\bar{f}\left(x^{-I}\right) \text {. }
$$


The domain, $D\left(S^{*}\right)$, of $S^{*}$ consists of those functions in $L^{2}(G)$ which are square integrable with respect to right Haar measure: $\Delta\left(x^{-1}\right) d x$. We will write $\Delta^{ \pm \frac{1}{2}}$ for the operator of multiplication by $\Delta(x)^{ \pm \frac{1}{2}}$. It is also easy to verify that the operators $R_{x}(x \in G)$ defined on $L^{2}(G)$ by

$$
\left(R_{x} f\right)(y)=f(y x)
$$

are bounded. Finally, convolution of functions will be written $f * g$ and the inner product for all Hilbert spaces will be conjugate linear in the first variable.

For more information on reproducing kernels on groups the reader is referred to KreYn [9, 10] and Carey [1]. We remark here that reproducing kernels are just positive definite functions in another guise. For an elementary account of the unimodular theory of square integrable representations the reader is referred to Chapter VII of Gaal [4].

\section{Preliminary results}

Let $\pi$ be a cyclic continuous unitary representation of $G$ in a Hilbert space $H$, with cyclic vector $v,\|v\|=1$. Define $\phi: G \rightarrow C$ by $\phi(x)=\langle v, \pi(x) v\rangle$. Let us suppose that $\phi$ is square integrable with respect to left Haar measure on $G$. Then by a result of Godement [7] (see also Theorem 13.8 .6 of Dixmier [2]) there is a $\psi \in L^{2}(G)$ with $\psi$ positive definite such that $\phi=\bar{\psi} * \bar{\psi}$. Now

$$
\begin{aligned}
\phi(y) & =\int \bar{\psi}(x) \bar{\psi}\left(x^{-1} y\right) d x \\
& =\left\langle\psi, L_{y} \psi\right\rangle,
\end{aligned}
$$

where $\langle$,$\rangle denotes the inner product in L^{2}(G)$ and $y \rightarrow L_{y}$ the left regular representation of $G$. Hence the map $W: \pi(y) v \rightarrow L_{y} \psi$ extends to a unitary equivalence of $\pi$ with a subrepresentation of $L$ (cf. Lemma 14.1.1 of [2]).

Conversely if $H \leq L^{2}(G)$. is invariant under $L$ then let $P$ be the projection onto $H$. Since the continuous functions of compact support, $C_{0}(G)$, are dense in $L^{2}(G), P C_{0}(G)$ is dense in $H$. We show below that 
for all $g \in C_{0}(G)$ the function

$$
x \rightarrow\left\langle P g, L_{x}^{-1} P g\right\rangle
$$

is square integrable. Hence we may define an irreducible continuous unitary representation $\pi$ of $G$ to be square integrable if there exists a vector $v \in H$ such that the coefficient function

$$
x \rightarrow\langle v, \pi(x) v\rangle
$$

is square integrable. Then by the above (as in the unimodular case) it follows that the square integrable representations of $G$ are just the irreducible subrepresentations of the left regular representation. So we need to prove

LEMMA 2.1. If $g \in C_{0}(G)$ then the function $x \rightarrow\left\langle P g, L_{x}^{-1} P g\right\rangle$ is square integrable.

$$
\text { Proof. Since }\left\langle P g, L_{x}^{-1} P g\right\rangle=\left(P g * S^{*} g\right)(x) \text { we need to show that }
$$

$P g * S^{*} g$ is in $L^{2}(G)$. This will follow by the Riesz representation theorem if we can show that the linear functional on $L^{2}(G)$ given by

$$
Z(h)=\int\left(P g * S^{*} g\right)(y) h(y) d y, h \in L^{2}(G),
$$

is bounded. We have

$$
\begin{aligned}
\left|\int \overline{g(x)} \int P g(y x) h(y) d y d x\right| & \leq \int \overline{g(x)}\left\|R_{x} P g\right\| d x\|h\|_{2} \\
& \leq\left\|\Delta^{-\frac{3}{2}} g\right\|_{1}\|P g\|_{2}\|h\|_{2},
\end{aligned}
$$

using the fact that $\left\|R_{x} f\right\|=\Delta(x)^{-\frac{1}{2}}\|f\|$. Now for $h \in C_{0}(G)$ it follows from Fubini's theorem that

$$
Z(h)=\int \overline{g(x)} \int P g(y x) h(y) d y d x,
$$

and therefore the above inequality shows that $l$ extends to a continuous linear functional on $L^{2}(G)$; whence $P g * S^{*} g \in L^{2}(G)$. 


\section{Reproducing kernels}

Let $\pi$ be an irreducible subrepresentation of the left regular representation of $G$ acting on $H_{\pi}$. Let $v \in H_{\pi}$ be such that the coefficient $\phi: x \rightarrow\langle v, \pi(x) v\rangle$ is square integrable and suppose that $\|v\|=1$. Then by the result of Godement quoted previously $\phi=\bar{\psi} * \bar{\psi}$ for some square integrable positive definite function $\psi$. Let $H$ be the closure of the linear span of $\left\{L_{y} \psi \mid y \in G\right\}$. Then the restriction of $L$ to $H$ is unitarily equivalent to $\pi$. We show that $B$ is a reproducing kernel Hilbert space.

Following Dixmier [2] we define the operator $R_{\psi}$ by

$$
R_{\psi} f=L_{f} \psi, f \in C_{0}(G)
$$

Now $R_{\psi}$ is not necessarily bounded; nevertheless by the positive definite property of $\psi, R_{\psi}$ admits a Friedrich's extension which is positive and self-adjoint. Further $R_{\psi}$ commutes with the operators $L_{x}$ for all $x \in G$.

We denote the spectral decomposition of $R_{\psi}$ by

$$
R_{\psi}=\int_{0}^{\infty} \lambda d E_{\lambda} .
$$

The spectral projections $E_{\lambda}$ commute with the operators $L_{x}$ for all $x \in G$, and we define $\psi_{\lambda}=E_{\lambda} \psi$ and $H_{\lambda}=E_{\lambda} H$. It follows as in the proof of Theorem 17 of Godement [7] that $\psi_{\lambda}$ is continuous and positive definite. A short calculation yields the relation

$$
R\left(\psi_{\lambda}\right)=R(\psi) E_{\lambda}
$$

whence $R\left(\psi_{\lambda}\right)$ is bounded. (Compare this paragraph with the proof of Theorem 13.8 .6 of [2].)

Now choose $\lambda_{0}$ so that $E_{\lambda_{0}} \neq 0$. Then it is not difficult to see that ${ }_{\lambda_{0}}$ (which is invariant under $L$ ) can have no non-zero, proper, 
closed $L$ invariant subspaces (by the irreducibility of $L$ restricted to $H$ ). If $g \in L^{2}(G)$ then

$$
R\left(\psi_{\lambda_{0}}\right) g(x)=\left\langle\psi_{\lambda_{0}}, L_{x}^{-1} g\right\rangle
$$

implying that if $g \in H_{\lambda_{0}}^{\perp}$ then $R\left(\psi_{\lambda_{0}}\right) g=0$; whence $R\left(\psi_{\lambda_{0}}\right) H_{\lambda_{0}} \subseteq H_{\lambda_{0}}$. Now since $R\left(\psi_{\lambda_{0}}\right)$ is bounded and commutes with $L$ restricted to $H_{\lambda_{0}}$, it must be a multiple of the identity on ${ }_{\lambda_{0}}$. Putting this together we have

$$
R\left(\psi_{\lambda_{0}}\right)=P_{\lambda_{0}} / d_{\lambda_{0}}
$$

where $P_{\lambda_{0}}$ is the orthogonal projection onto ${ }_{\lambda_{0}}$ and $d_{\lambda_{0}}$ is a positive constant.

If $\lambda>\lambda_{0}$ then $E_{\lambda} \geq E_{\lambda_{0}}$ and so $H_{\lambda} \geq H_{\lambda_{0}}$. But $H_{\lambda}$ can contain no invariant subspaces whence $H_{\lambda}=H_{\lambda_{0}}$. Using the same analysis as above we have $R\left(\psi_{\lambda}\right)=P_{\lambda_{0}} / d_{\lambda}$ and hence

$$
R(\psi) E \lambda_{\lambda} \bar{\lambda}_{0}=P_{\lambda_{0}} E_{\lambda_{0}} / d_{\lambda}=R(\psi) E_{\lambda_{0}}=P_{\lambda_{0}} / d_{\lambda_{0}}
$$

This implies that $d_{\lambda}=d_{\lambda_{0}}$. Hence for $\lambda>\lambda_{0}, R(\psi) E_{\lambda}=R(\psi) E_{\lambda_{0}}$ and using the fact that as $\lambda+\infty, E_{\lambda}$ converges strongly to the identity we have

$$
R(\psi)=P_{\lambda_{0}} / d_{\lambda_{0}}
$$

Now

$$
\left(a_{\lambda_{0}} R(\psi)\right)^{2}=a_{\lambda_{0}} R(\psi)
$$

implies that

$$
d_{\lambda_{0}} \psi * d_{\lambda_{0}} \psi=d_{\lambda_{0}} \psi=d_{\lambda_{0}} P_{\lambda_{0}} \psi=d_{\lambda_{0}} \psi_{\lambda_{0}}
$$


Hence $\psi_{\lambda_{0}}=\psi \in H \cap H_{\lambda_{0}}$ and so $H_{\lambda_{0}}=H$. We have almost proved

THEOREM 3.1. Let $\pi$ be an irreducible subrepresentation of the left regular representation of $G$. Let $\phi$ be a square integrable coefficient of $\pi$. Then there is a constant $d_{\phi}$ such that right convolution by $\psi=d_{\phi}^{2-}$ defines $\omega_{E}$ - ection, $R_{\psi}$ in $L^{2}(G)$. The space $R_{\psi} L^{2}(G)$ is a reproducing kernel Hilbert space with kemel $x, y \rightarrow \psi\left(x^{-1} y\right)$ and the restriction of $L$ to $R_{\psi} L^{2}(G)$ carries a representation equivalent to $\pi$.

Proof. Given $\phi$ one defines $\psi_{1}$ via the result of Godement [7]; that is, by the equation $\phi=\bar{\psi}_{1} * \bar{\psi}_{1}$. Then we saw above that there is a constant $d_{\phi}$, say, such that $d_{\phi} R\left(\Psi_{1}\right)$ is a projection. Since this implies that $d_{\phi} \psi_{1} * d_{\phi} \psi_{1}=d_{\phi} \psi_{1}$ we have $\psi_{1}=d_{\phi} \bar{\phi}$. So we define $\psi=d_{\phi}^{2} \bar{\phi}$, and $R(\psi)$ is then a projection. The continuity of elements of $R_{\psi} L^{2}(G)$ follows from the continuity of $\phi$, and the reproducing property of the function

$$
x, y \rightarrow \psi\left(x^{-1} y\right)=\psi_{x}(y)
$$

follows from

$$
\begin{aligned}
\left(R_{\psi} f\right)(x) & =\left\langle L_{x} \psi, f\right\rangle=\left\langle\psi_{x}, f\right\rangle \\
& =f(x) \text { if } f \in R_{\psi} L^{2}(G) .
\end{aligned}
$$

REMARK 3.2. The constant $d_{\phi}$ may also be characterized by the relation

$$
\|\bar{\phi}\|^{2}=\phi * \phi(e)=\bar{\psi}_{1} * \bar{\psi}_{1}(e) / d_{\phi}^{2}=1 / d_{\phi}^{2} .
$$

\section{The orthogonality relations}

To begin with we suppose that $R_{\psi} L^{2}(G)$ is an irreducible subspace of $L^{2}(G)$ with reproducing kernel $x, y \rightarrow \psi\left(x^{-1} y\right)$. Observe that 
$D\left(S^{*}\right) \cap R_{\psi} L^{2}(G)$ contains the vectors $L_{x} \psi=\psi_{x}$ since $S^{*} \psi_{x}=R_{x} \psi$ is in $L^{2}(G)$. Noting that the linear span of $\left\{\psi_{x} \mid x \in G\right\}$ is dense in $R_{\psi} L^{2}(G)$ we conclude that $R_{\psi} L^{2}(G) \cap D\left(S^{*}\right)$ is dense in $R_{\psi} L^{2}(G)$.

LEMMA 4.1. For all $f \in D\left(S^{*}\right) \cap R_{\psi} L^{2}(G)$ and $f^{\prime} \in R_{\psi} L^{2}(G)$, the function $c_{f f^{\prime}}: x \rightarrow\left\langle f, L_{x}^{-1} f^{\prime}\right\rangle$ is square integrable.

Proof. The function $x \rightarrow\left\langle f, L_{x}^{-1} \psi\right\rangle=\bar{f}\left(x^{-1}\right)=S^{*} f(x)$ is, by hypothesis, square integrable for every $f \in D\left(S^{*}\right) \cap R_{\psi} L^{2}(G)$. Let $D$ be the subspace of all $f^{\prime} \in R_{\psi} L^{2}(G)$ such that $c_{f f}$ is square integrable. $D$ contains a non-zero vector $\psi$ and is invariant under $L$, whence $D$ is dense in $R_{\psi} L^{2}(G)$.

Now following Gaal [4] we define $T_{f}: D+L^{2}(G)$ by

$$
\left(T f^{\prime}\right)(x)=\left\langle f, L_{x^{-1}} f^{\prime}\right\rangle
$$

It now follows as in Proposition VII.1.4 of Gaal [4] that $T_{f}$ is bounded on $D$ and hence $D=H$, proving the result.

By using the argument of the above lemma, it is clear that if $x \rightarrow\left\langle f, L_{x^{-1}} f^{\prime}\right\rangle$ is square integrable for some $f^{\prime} \in R_{\psi} L^{2}(G)$ then it is square integrable for all $f^{\prime} \in R_{\psi} L^{2}(G)$ and hence in particular that $x \rightarrow\left\langle f, L_{x^{-I}} \psi\right\rangle$ is square integrable. Thus $f \in D\left(S^{*}\right)$ and so the above lemma specifies all the square integrable functions $c_{f f}$.

PROPOSITION 4.2. If $f_{i}, g_{i}, i=1,2$, are elements of the irreducible subspace $R_{\psi} L^{2}(G)$ of $L^{2}(G)$, then there is a constant $d_{\psi}$ such that

$$
\left.\int_{G} \overline{\left\langle f_{1}, L_{x} f_{2} \times\right.} g_{1}, L_{x} g_{2}\right\rangle d x=\left\langle S^{*} g_{2}, S^{*} f_{2} \times g_{1}, f_{1}\right\rangle / d_{\psi}
$$


whenever $f_{2}, g_{2} \in D\left(S^{*}\right)$.

Proof. Following the proof of Proposition VII.1.29 of Gaal [4] we observe firstly that the integral has the form

$$
\left\langle T_{f_{2}} f_{1}, T_{g_{2}} g_{1}\right\rangle_{L^{2}(G)},
$$

where $T_{f_{2}},{ }^{T} g_{2}$ are the operators introduced in the preceding lemma. Now $T_{g_{2}} * T_{f_{2}}: R_{\psi} L^{2}(G) \rightarrow R_{\psi} L^{2}(G)$ and it is not difficult to show that

$T_{g_{2}} * T_{f_{2}}$ commutes with the action of $L$ in $R_{\psi} L^{2}(G)$. Hence $T_{g_{2}} * T_{f_{2}}$ is a constant multiple, say $k\left(g_{2}, f_{2}\right) I$ of the identity operator.

So we have the value of the integral as

$$
k\left(g_{2}, f_{2}\right)\left\langle f_{1}, g_{1}\right\rangle
$$

To find $k\left(g_{2}, f_{2}\right)$ we set $f_{1}=g_{1}=\psi$ and obtain

$$
\begin{aligned}
\int_{G}\left\langle f_{2}, \psi_{x^{-1}}\right\rangle\left\langle\psi_{x^{-1}}, g_{2}\right\rangle d x & =\left\langle S^{*} g_{2}, S^{*} f_{2}\right\rangle \\
& =k\left(g_{2}, f_{2}\right)\langle\psi, \psi\rangle .
\end{aligned}
$$

Hence

$$
\begin{aligned}
k\left(g_{2}, f_{2}\right) & =\left\langle s^{*} g_{2}, s^{*} f_{2}\right\rangle /\langle\psi, \psi\rangle \\
& =\left\langle S^{*} g_{2}, s^{*} f_{2}\right\rangle / d_{\psi}
\end{aligned}
$$

where $d_{\psi}$ is defined to be $\|\psi\|^{2}$.

To connect this proposition with the formulation of the orthogonality relations given in [3], [8], [11] we follow Phillips [11]. Consider the map $\Delta^{-\frac{1}{2}}: D\left(S^{*}\right) \rightarrow L^{2}(G)$ defined as in the introduction. Since $\Delta^{-\frac{1}{2}}$ is closed its restriction to $D=D\left(S^{*}\right) \cap R_{\psi} L^{2}(G)$ is also closed as an operator from $D$ into the completion (in $L^{2}(G)$ ) of $\Delta^{-\frac{2}{2}} D$. We may therefore define the adjoint 


$$
\left(\Delta^{-\frac{1}{2}}\right)^{*}: \Delta^{-\frac{1}{2}} D \rightarrow D
$$

Now. let $T: D \rightarrow D$ be given by

$$
T=\left(\left(\Delta^{-\frac{3}{2}}\right)^{*} \Delta^{-\frac{1}{2}}\right)^{\frac{1}{2}} /\|\psi\|^{2} .
$$

It follows that

$$
\left\langle T f, T f^{\prime}\right\rangle=\left\langle S^{*} f^{\prime}, S^{*} f\right\rangle /\|\psi\|^{2}
$$

Furthermore

$$
D(T)=D\left(S^{*}\right) \cap R_{\psi} L^{2}(G) \text { as }\left\|\Delta^{-\frac{1}{2}} f\right\|=\left\|S^{*} f\right\| \text {. }
$$

THEOREM 4.3. Let $\pi$ be an irreducible representation of $G$ in $H_{\pi}$ with a square integrable coefficient $\phi: x \rightarrow\langle v, \pi(x) v\rangle$, where $v \in H_{\pi}$ and $\|v\|=1$. Let $W: H_{\pi} \rightarrow L^{2}(G)$ be the unitary equivalence of $\pi$ with the restriction of $L$ to $R_{\psi} L^{2}(G)$, where $\psi=\bar{\phi} /\|\phi\|^{2}$. Let $T_{\pi}=W^{-1} T W$; then for all $u_{1}, v_{1} \in D\left(T_{\pi}\right)$ and $u_{2}, v_{2} \in H_{\pi}$,

$$
\left.\int\left\langle\pi(x) u_{1}, u_{2}\right) v_{2}, \pi(x) v_{1}\right) d x=\left\langle T_{\pi} u_{1}, T_{\pi} v_{1} \times v_{2}, u_{2}\right\rangle \text {. }
$$

Proof. Combining Proposition 4.2 and equation (4.1) we obtain the result.

REMARKS 4.4. (i) $T_{\pi}$ is the only positive operator defined on $W D(T)$ which satisfies (4.2) for every $u_{2}, v_{2} \in H_{\pi}$ and $u_{1}, v_{1} \in W D(T)$.

(ii) Unlike the unimodular case, the "formal dimension" $d_{\psi}=\psi(e)=\|\psi\|^{2}$ depends not only on the equivalence class of $\pi$ but also appears to depend on the choice of minimal projection $R_{\psi}$; that is, on the realization of the square integrable representation chosen.

(iii) In the case of separable unimodular $G$, Segal [12] introduced the projections on $L^{2}(G)$ of the form $R_{f}$ where $f \in L^{2}(G)$. He called them finite projections and showed that every projection in the commuting algebra of $L$ is the least upper bound of the finite projections it bounds. This enabled him to demonstrate that in the central decomposition 
of the commuting algebra, no type III factors occur. It would be interesting to investigate to what extent similar results are true in the non-unimodular case.

\section{References}

[1] A.L. Carey, "Group representations in reproducing kernel Hilbert spaces" (Preprint, Department of Mathematical Physics, University of Adelaide, Adelaide, 1975).

[2] Jacques Dixmier, Les $\mathrm{C}^{*}$-algèbres et leurs représentations (GauthierVillars, Paris, 1964).

[3] M. Duflo and Calvin C. Moore, "On the regular representation of a nonunimodular locally compact group", J. Functional Analysis 21 (1976), 209-243.

[4] Steven A. Gaal, Linear analysis and representation theory (Die Grundlehren der mathematischen Wissenschaften, 198. SpringerVerlag, Berlin, Heidelberg, New York, 1973).

[5] Roger Godement, "Sur les relations d'orthogonalité de V. Bargmann. I. Résultats préliminaires", C.R. Acad. Sci. 225 (1947), $521-523$.

[6] Roger Godement, "Sur les relations d'orthogonalité de V. Bargmann. II. Démonstration générale", C.R. Acad. Sci. 225 (1947), 657-659.

[7] Roger Godement, "Les fonctions de type positif et la théorie des groupes", Trons. Amer. Math. Soc. 63 (1948), 1-84.

[8] Adam Kleppner and Ronald L. Lipsman, "The Plancherel formula for group extensions II", Ann. Sci. Ecole. Norm. Sup. (4) 6 (1973), 103-132.

[9] M.G. Kreǐn, "Hermitian-positive kernels on homogeneous spaces. I", Amer. Math. Soc. Tronsl. (2) 34 (1963), 69-108.

[10] M.G. Kreīn, "Hermitian-positive kernels on homogeneous spaces. II", Amer. Math. Soc. Transi. (2) 34 (1963), 109-164. 
[11] John Phillips, "A note on square-integrable representations", $J$. Functional Analysis 20 (1975), 83-92.

[12] I.E. Segal, "An extension of Plancherel's formula to separable unimodular groups", Ann. of Math. (2) 52 (1950), 272-292.

Department of Mathematical Physics, University of Adelaide,

Adelaide,

South Australia. 